\title{
The Representation of Spatial Context by Emojis within Location based Social Media
}

\author{
Eva Hauthal ${ }^{\mathrm{a}, *}$, Alexander Dunkel ${ }^{\mathrm{a}}$, Dirk Burghardt ${ }^{\mathrm{a}}$ \\ a Institute of Cartography, Technische Universität Dresden, Germany; firstname.lastname@tu-dresden.de \\ * Corresponding author
}

Keywords: Emojis, Location-Based Social Media, Context

\begin{abstract}
:
The presented study investigates to which extent emojis included in georeferenced social media posts reflect the user's surroundings. From our perspective, emojis represent language-independent indicators of the user's context in terms of performed activities related to the location of the post as well as objects being perceived in the physical environment. Due to the growing number of available emojis and the even more growing use of them for communication, this is an insufficiently explored potential of these seemingly infantile little pictograms so far. Though, other research shows that emojis reflect or even complement the content of a photo, that they provide information about emotions, serve as decoration or lexical units, and thus say more than the associated text when considered standing alone. Our aim is to identify a correlation between the emojis used and the location of the respective social media post.
\end{abstract}

Posts on the topic of sunrise and sunset on the platform Instagram were used as a vehicle for the study. The choice of this topic is driven by the fact that this is an event that is recurring on a daily basis, widely noticed, appreciated and shared on social media. The total of 11 million posts in this global Instagram dataset, stored in a privacy-aware data format based on the Hyperloglog approach, were subdivided into two sub-datasets, relating to either sunrise or sunset. The typicality measure was applied to the emojis contained within them (Hauthal et al. 2021). This measure indicates whether an emoji within a sub-dataset is typical or atypical compared to the total dataset. Emoji typicality was calculated at different scale levels (global, national, etc.), i.e., using different total and sub-datasets in each case.

At all scale levels, it can be seen that the emojis determined as most typical provide significantly more information about the context, i.e., the user's surroundings and the activities performed in connection with sunrise and sunset, than the most frequently used ones. The latter only show that the event photographed is related to sun, whereas the former show that sunrise is observed while jogging, drinking coffee or during mountain hikes, whereas sunset is commonly witnessed while having a sundowner in the city or at the beach.

Some of the emojis calculated as typical were attributed as location-specific. Location-specific emojis are demarcated by the fact that they were selected depending on the location of the social media post and show an object of the physical environment. At all scale levels studied, these emojis represent either a montane, maritime, or urban environment or the prevailing tree vegetation. The more detailed the scale level, the more particular the location-specific emojis are. For example, in Italy, which was chosen as the national scale level, volcanoes were among the location-specific emojis, which corresponds to actual conditions in Italy. On a global scale, this was not the case, as this is of course a much more general view. By visualising the location-specific emojis used in the dataset in maps, patterns emerge that correspond to the real situation. Moreover, the calculation of correlation coefficients between the typicality of locationspecific emojis and various representative geographical parameters (elevation span, coast ratio, urban population, forest coverage) reveals a consistently positive correlation at the analysed scale levels.

Accordingly, it can be concluded that emojis reflect the actual circumstances and thus the context and surrounding of the user. This suggests that they can be rich source of data also on another level and can provide multifaceted information in a language-independent and efficient way that cannot be obtained completely, not at all or only with much effort by purely text-based analyses.

\section{References}

Hauthal, E., Dunkel, A., and Burghardt, D., 2021. Emojis as Contextual Indicants in Location-Based Social Media Posts. ISPRS International Journal of Geo-Information 10, no. 6: 407. https://doi.org/10.3390/ijgi10060407 